\title{
UN'INDAGINE NELLA RETORICA: DALLA VULNERABILITÀ SOCIALE DI ZOLA ALLA DEUMANIZZAZIONE DI KAFKA
}

\author{
VIRGINIA ZAMBRANO ${ }^{1}$
}

\begin{abstract}
RiASSUNTO: Per i giuristi, mettere in relazione diritto e letteratura è importante. La congiunzione dei due elementi permette di corrodere la dogmatica giuridica, desacralizzare e restituire il diritto alla propria misura e alla misura dell'etica. Ciò che, infatti, la letteratura insegna, rispetto all'assetto astrattamente codificato del diritto, è l'inesistenza di leggi generali nell'esperienza vissuta. Ne consegue che le condanne, come le assoluzioni, sono inevitabilmente imperfette: siamo tutti in qualche modo colpevoli, ma anche, secondo diverso angolo di lettura, tutti innocenti. E se i decreti di colpevolezza assoluta appaiono inadeguati nelle opere di Proust, Musil e Hofmannsthal, non diversa è la situazione che si respira nell'universo di Kafka e di Zola. A questa prima decostruzione del diritto se ne aggiunge una seconda, ancor più radicale, relativa non più ai suoi limiti, ma alla stessa essenza del diritto. Non solo il sistema normativo non riesce ad incasellare nelle proprie griglie una realtà umana sfuggente ad un ordine prefissato, ma, tutt'altro che situarsi al polo opposto della violenza criminale, confina ambiguamente con essa. In questo senso, l'intersezione fra diritto e letteratura insegna che le situazioni individuali vanno sempre calate in quel caleidoscopio sociale, culturale, storico, economico, che condiziona i nostri atti non meno della nostra volontà.
\end{abstract}

Parole Chiave: visione del diritto: Kafka e Zola; decostruzione e limiti del diritto; giustizia come forma di esperienza.

Nulla gli uomini aborriscono come il giudizio, quest'atto senza scopo che hanno messo al centro della loro esistenza. Ciascuno è intimamente innocente: e il vero innocente non è colui che viene assolto, bensì colui che passa nella vita senza giudizio (Satta, 1968, p. 10).

1. Il "ventre della balena" era, per Benedetta Tobagi (2009), il Palazzo di Giustizia, in cui si celebrava il processo ai terroristi rossi che avevano ucciso

\footnotetext{
1 Dottorato presso l'Università degli Studi di Napoli "Federico II" (Italia). Professore Ordinario di Diritto Privato Comparato nell'Università di Salerno (Italia). Salerno, Italia. E-mail:vzambrano@unisa.it
} 
suo padre, il giornalista Walter Tobagi. In quel "ventre", dove la macchina della giustizia svolge le sue codificate procedure, l'uomo comune si immerge provando spesso un senso di disorientata estraneità.

Ad avvicinare la matassa della vita all'apparente astrattezza del diritto può concorrere il mondo della letteratura. Sembra trattarsi di due realtà diverse. Un solco profondo sembra separare la fluidità senza confini della letteratura e la rigidità dell'ordine giuridico.

E si. Perché l'ordine giuridico è chiamato costantemente a tracciare linee di demarcazione tra il giusto e l'ingiusto e quindi, sia pure trasversalmente o tangenzialmente, tra il bene e il male. La relazione tra diritto e letteratura diventa così fonte di numerose suggestioni per il giurista. Si è in presenza di un diverso modo di guardare al dialogo fra diritto e realtà sociale. Un dialogo che muta, si espande, poiché prende in considerazione costituenti diverse senza arrestarsi al mero dato economico $\mathrm{o}$ sociale. Se è vero che, tradizionalmente, obiettivo del giurista è interrogarsi sulle condizioni che conducono alla "costruzione" del dato normativo nonchè andare alla ricerca delle ragioni che ne sono alla base; è del pari indubbio che, oggi, questa osservazione si arricchisce della necessità di considerare altri elementi. L'analisi del giurista ne esce impreziosita. Non si tratta più di verificare il grado di corrispondenza dell'architettura giuridica a quella sociale e, tanto meno, andare alla ricerca di un suo maggiore o minor grado di utilità sociale. Il diritto, nella sua apparente neutralità e impersonalità, pretende offrire una risposta generale in grado di "servire" bene le esigenze di costruzione dell'ordine sociale.

E tuttavia c'è qualcosa che trascende la verità giudiziaria, che solo la letteratura può cogliere (Forti et al., 2012, 2014)². È la zona d'ombra della

\footnotetext{
"In un apologo diffuso in ambienti scientifici si racconta di un poliziotto che in una notte oscura si imbatte in un ubriaco. Questi sta inginocchiato alla ricerca di qualcosa sotto il lampione. Alla domanda dell'agente su cosa sta facendo, l'ubriaco risponde che sta cercando le chiavi di casa che dice di avere perso laggiù indicando in direzione dell'oscurità. Il poliziotto gli chiede: «Ma perché se le ha perse laggiù le cerca sotto il lampione?» L'ubriaco risponde: «Perché c'è molta più luce.» Riferito al mondo dei legislatori, dei criminologi e dei penalisti l'apologo può essere interpretato nel senso di suonare come un invito rivolto ai medesimi, senza per questo ritenerli ubriachi, a non limitare studi e ricerche nell'ambito delle categorie del diritto, cioè a dove arriva la luce del lampione, ma a spingersi a cercare dove è buio, nell'oscurità dei comportamenti degli autori dei reati, là dove giace nascosta la chiave smarrita della giustizia. Un apologo, se
} 
comprensione umana, emotiva sentimentale, poetica. E questo accade proprio perché, come asserisce Claudio Magris (2006), la letteratura né dà voti di condotta alla vita, che scorre al di qua e al di là del bene e del male, né ha l'am-bizione di giudicare, tanto meno secondo le formalizzazioni del diritto. Pur quando mette in scena le dinamiche processuali, si avventura piuttosto verso territori oscuri che l'iter giudiziario non può conoscere: se si rileggono con questo sguardo i grandi testi rivolti alla questione della legge, si ha l'impressione che essi siano in grado di rivelare qualcosa del diritto che questo, dall'interno del proprio linguaggio, non arriva ad afferrare l'ombra che circonda la sua luce o il punto oscuro in cui essa rischia di spegnersi. Se opere come il Mercante di Venezia e l'Otello di Shakespeare rivelano il pregiudizio razziale, che sottende il giudizio di condanna, i romanzi di Defoe, da Lady Roxana a Moll Flanders, mostrano, dietro il delitto, una condizione di estremo bisogno che in qualche modo ne eccede la rilevanza penale, aprendo uno squarcio nel formalismo della legge3.

non proprio ottimista, che suona tuttavia da stimolo alla possibilità che i grandi testi della letteratura offrano un terreno di indagine alla comprensione della giustizia" (Camerana, 2013, p. 5).

Il movimento chiamato "Diritto e letteratura" prende avvio negli Stati Uniti con la pubblicazione nel 1908 - nel clima della "rivolta contro il formalismo" - di A List of Legal Novels di J. Wigmore (1908). In essa vengono selezionati pezzi di narrativa interessati a tematiche giuridiche col dichiarato fine di diffondere le opere letterarie che testimoniano i valori giuridici fondamentali della cultura americana per informare ad essi i giuristi. Negli anni successivi vengono pubblicate altre opere di questo genere, che consolidano la convinzione che la letteratura contribuisca a formare la coscienza etica di avvocati e giuristi e, per questa via, la prospettiva giusletteraria entra nell'insegnamento universitario. Nel 1925 col saggio Law and Literature, B. Cardozo (1925) prefigura la possibilità, da un lato, di leggere ed interpretare le sentenze come esempi di letteratura, o meglio di scrittura letteraria, e, dall'altro, di cogliere, attraverso la rappresentazione letteraria, il contesto in cui si svolge l'esperienza giuridica, il law in action contrapposto al law in books. Negli stessi anni, in Italia, numerose sono le opere che danno spazio a diritto e letteratura; senza pretesa alcuna di completezza, si ricordino: G. Brunelli, che nel saggio del 1906 Il fatto illecito e il fatto immorale di fronte al diritto positivo, analizza il Mercante di Venezia di Shakespeare per argomentare in tema di rapporti tra diritto positivo, interpretazione giuridica e morale; P. Cogliolo, che nel saggio del 1940 La Lingua giuridica, si sofferma a valutare la bellezza delle espressioni del diritto e il ricorrere delle figure retoriche nel linguaggio normativo; A. Ascoli e C. Levi, che nel 1914 pubblicano il saggio Il diritto privato nel teatro contemporaneo francese e italiano; P. Calamandrei, che nel 1924 nel saggio Le lettere e il processo civile scrive "dalla lettura di certe pagine di romanzi, nelle quali si descrivono con linguaggio profano i congegni della giustizia in azione, è assai spesso possibile trarre un'idea precisa, meglio che da una critica fatta in gergo tecnico e in stile cattedratico, del modo in cui la realtà reagisce sulle leggi e della loro inadeguatezza a raggiungere nella vita pratica gli scopi per i quali il legislatore crede di averle create" (1924, p. 204); L. Tumiati, che nel saggio del 1927 La poesia nel diritto si focalizza sulla dimensione artistica del diritto; R. Vacca, che nel libro Il diritto sperimentale del 1923 sostiene che l'opera letteraria non si limita a descrivere il reale, ma rappresenta la psicologia dei personaggi, così che le opere di Rabelais, Dickens, Balzac e Tolstoj spiegano "certi modi di agire e di pensare inerenti alla natura umana assai meglio 
Delitto e castigo di Dostoevskij, poi, attraverso la tormentata vicenda di Raskol'nicov, spinge la domanda sulla colpa ai suoi estremi confini - in quella zona indistinta ove bene e male, orrore e compassione, si intrecciano in una vertiginosa spirale4.

Porre in relazione i due universi, congiungere i due elementi, dunque, può risultare produttivo per gli specialisti del diritto. L'obiettivo è quello di corrodere la dogmatica giuridica. Desacralizzare e restituire il diritto alla propria misura e alla misura dell'etica. Riportare alla luce, scrive Maurice Maeterlinck, "sulla punta delle nostre dita pallide, certi meravigliosi tesori che, in una caverna, giacciono nel mare profondo" (1989, p. 55)5. Ciò che la letteratura insegna, rispetto all'assetto astrattamente codificato del diritto, è che nell'esperienza vissuta non esistono leggi generali, giacché i casi della vita sono sempre singolari e irripetibili. Perciò le condanne, come le assoluzioni, risultano inevitabilmente imperfette: siamo tutti in qualche modo colpevoli, ma anche, secondo diverso angolo di lettura, tutti innocenti. E così i decreti di colpevolezza assoluta appaiono inadeguati nelle opere di Proust, Musil e Hofmannsthal: le situazioni individuali vanno sempre calate in quel caleidoscopio sociale che condiziona i nostri atti non meno della nostra volontà.

2. A questa prima decostruzione del diritto se ne aggiunge una seconda, ancor più radicale, relativa non più ai suoi limiti, ma alla sua stessa essenza.

di qualche vecchio trattato di filosofia del diritto, ed anche di qualche moderno manuale di psicologia giudiziaria” (1923, p. 204). Cfr. altresì A. D’Amato, La letteratura e la vita del diritto, pubblicata nel 1936; F. Pergolesi, la cui ampia produzione sul tema comincia negli anni '20 con Il diritto nella letteratura (1927), ma trova definitivo sviluppo negli anni '40 e '5o con, in particolare, Diritto e giustizia nella letteratura moderna narrativa e teatrale (1949, riedito con aggiornamenti nel 1956).

4 Si ricordino le parole scritte da Henri-Louis Bergson in Les deux sources de la morale et de la religion: "che faremo noi se apprendessimo che per la salute del popolo, per l'esistenza stessa dell'umanità, ci fosse in qualche luogo un uomo, un innocente che è condannato a eterne torture? Noi vi consentiremo forse, a patto che un filtro magico ce lo facesse dimenticare, a patto che non ne sapessimo più nulla: ma se noi dovessimo saperlo, pensarci, dirci che quest'uomo è sottoposto ad atroci supplizi perché noi potessimo esistere, che questa è una condizione dell'esistenza in generale, ah no, piuttosto accettare che nulla più esista, piuttosto lasciar saltare il pianeta!” (1932, p. 41).

${ }_{5}$ "Il recupero del diritto nella letteratura porta alla scoperta di tesori nascosti, che tutti possono apprezzare cogliendo le potenzialità di un arricchimento non solo intellettuale, culturale e morale, ma anche professionale: sono i frutti editoriali di cui abbiamo bisogno per rendere anche migliore e più giusta la professione dei giuristi” (Danovi, 2014, p. 190). 
Non solo il sistema normativo non riesce ad incasellare nelle proprie griglie una realtà umana sfuggente ad un ordine prefissato. Il diritto non sembra collocarsi al polo opposto della violenza criminale, ma confinare ambiguamente con essa. I romanzi Il sospetto, Il giudice e il suo boia di Durrenmatt ne forniscono la più tesa rappresentazione: in essi coloro che vogliono affermare la giustizia procedono adoperando i medesimi metodi criminali che intendono punire. Ma se il desiderio di giustizia si trasforma in sete di vendetta, il giudice diventa giustiziere e questi cacciatore di prede. Siamo al punto in cui la giustizia distributiva, che attribuisce a ciascuno la sua pena, diviene volontà di infliggere il male, rendendo intercambiabili colpevole e vittima. Ed è quello che descrive Kafka.

Non già (e soltanto) perché i suoi protagonisti sono catturati nelle maglie della legge, ma perché (e soprattutto) la pena è presupposta alla colpa che dovrebbe punire. Nel suo parossistico mondo a rovescio, non è più la colpa a determinare la pena, ma questa a produrre quella. E' questa la funzione che il Nomos eredita dal mondo demonico che lo precede schiacciare la vita sulla nuda parete del destino, nella letteratura filosofica di Benjamin e di Camus. Le immagini kafkiane della giustizia bendata non perché imparziale, ma perché colpisce alla cieca le sue vittime - e della macchina che incide la norma trasgredita sulla carne del colpevole, nascono da questo orizzonte.

Ma si proceda per ordine.

La letteratura, dunque, nella prima accezione funzionale, come denuncia sociale. Ma la letteratura è anche espressione del modo in cui è percepita la realtà normativa. La domanda che sorge, allora, quando si leggono Zola e Kafka è: cosa li ha fatti pensare così?

La risposta involve un'investigazione nella retorica. Si tratta cioè di capire un discorso immaginando che tutti gli elementi siano imbevuti di significato. Nel caso di Zola e Kafka il filo conduttore è rappresentato dal fatto che la loro scrittura è fortemente influenzata dal lavoro che svolgono. Critico e giornalista Zola, assicuratore e burocrate Kafka, il loro lavoro si riflette nella scrittura e rende evidente il contrasto fra il lavoro moderno e il lavoro artistico.

In Kafka, la fusione tra modernità e burocrazia, riflette la sua doppia vita di assicuratore e di scrittore. È una fusione che fa emergere tutta 
l'intrinseca contraddittorietà dell'epoca moderna. Su questi percorsi si sviluppa un itinerario letterario unico che investe il rapporto fra diritto e legalità. In discussione, comunque, non è tanto la norma in quanto tale, ma le sue componenti psicologiche, antropologiche teleologiche.

In Zola, la scrittura deve restituire il senso della realtà sociale, senza finzioni. Il padre del romanzo sperimentale, raffigura le realtà più dolorose, squallide e degradate con la stessa oggettività di un patologo nell'analisi dei sintomi di una terribile malattia. Perché come il giornalista, lo scrittore non può che rappresentare la realtà nella sua crudezza. La scrittura è denuncia sociale. La vulnerabilità delle classi inferiori è l'oggetto della denuncia. Una condizione che si fa strumento nelle mani della borghesia che vuole dimostrare la fatalità delle distinzioni sociali. Il corpo sociale è un corpo povero, sofferente che non riesce a dialogare con le istituzioni.

In Germinale (Zola, 2005) è significativo il corteo urlante attraversa numerosi impianti minerari, li devasta per rendere impossibile l'estrazione del carbone, e infine arriva nei pressi delle case dei borghesi. Massa di disperati e forsennati rossi: vera e propria schiera di demoni insanguinati ${ }^{6}$.

Ma la vera causa dell'odio proletario non risiede nella perfidia della classe inferiore, priva di autentica dignità, intrinsecamente barbara e

\footnotetext{
Per la trama del celebre romanzo Germinale, l'autore si ispirò alle proteste operaie verificatesi nel giugno e nell'ottobre del 1869 nel dipartimento della Loira e in quello dell'Aveyron: in entrambi i casi, lo sciopero dei minatori venne schiacciato dall'esercito, che sparò sui lavoratori provocando, nel complesso, ventisette morti. Il titolo del romanzo dedicato ai minatori e alle loro lotte fu scelto dipoi con chiaro riferimento alla grande manifestazione popolare svoltasi a Parigi il $1^{\circ}$ aprile 1795 (12 germinale dell'Anno Terzo della Repubblica, secondo il calendario rivoluzionario). In quel giorno, una folla enorme invase la Convenzione al grido di "Pane, e Costituzione del " 93 ”: i popolani chiedevano che il governo tornasse ad assumere un atteggiamento simile a quello tenuto durante il Terrore da Robespierre e dai giacobini, i quali (pur non essendo socialisti, cioè accettando in linea teorica il concetto di proprietà privata) si erano preoccupati della miseria delle masse e avevano tentato di far sì che tutti i cittadini, a prescindere dal loro censo, potessero accedere alla gestione dello Stato, tramite il voto (suffragio universale). L'opera si conclude tragicamente: lo sciopero fallisce, molti operai sono stati uccisi, il manovale Stefano Lantier (incarnazione del gradualismo marxista) è costretto a fuggire, il tempo della rivoluzione proletaria, del socialismo e della completa uguaglianza appare indefinito, remoto, irraggiungibile. Epperò, Zola non è né rassegnato né disperato: la sua amara constatazione del fallimento della liberazione degli oppressi riguarda un solo specifico episodio, non già l'intera vicenda umana.
} 
violenta (come il criminologo torinese Lombroso pur sosteneva7). Essa risiede nell'ottusità secolare dei padroni, che hanno sottoposto a sfruttamento servile, a condizioni inumane e degradanti i lavoratori: "Sentiva che i veri colpevoli erano tutti; che tutto ciò era la conseguenza di una colpa collettiva, secolare. Oh, certo, dei bruti, gli autori di quello scempio; ma dei bruti che non sapevano leggere e che crepavano di fame" (Zola, 2005, p. 216). Condizioni che avevano portato Parigi alle barricate del 1848.

Il degrado economico e la miseria generano inesorabilmente alcolismo, criminalità, propensione alla violenza, devastazione fisica e morale e sottolineano quanto forti siano le diseguaglianze sociali.

Narrata è la banalità della vita quotidiana, le passioni morbose - in Teresa Raquin di Zola (2009a) - che sfiorano il crimine e la patologia, la condizione delle classi sociali e tutta la miseria del proletariato. L'epica dei sobborghi tiene la scena dell'Assommoir (Zola, 2009b), quel margine urbano che non ha più nulla dell'aria di campagna ed è tuttavia condannato ad essere escluso dalle comodità della vita cittadina.

L'obiettivo di Zola è la descrizione di quella tassonomia sociale che sintetizza, in Francia, il secondo impero. Emerge con estrema chiarezza la concezione deterministica del naturalista francese: poste determinate condizione, gli eventi si produrranno con inflessibile necessità. La rivoluzione sarà inevitabile se le condizioni di vita e di lavoro di questi demoni, uomini disperati, non animali feroci privi di senno e razionalità, non vengono migliorate in modo radicale e tempestivo dalla borghesia e dal governo. Come ogni altra reazione chimica, essa proseguirà in modo necessario solo se la situazione permane immutata. Esiste una forte tensione fra una top-down strategy, basata sulle scelte politiche ed

\footnotetext{
Pur condividendo con Cesare Lombroso alcuni postulati di partenza, che provengono dalla comune appartenenza al clima culturale positivista, le posizioni dello scrittore naturalista francese e quelle del criminologo torinese non potrebbero essere più diverse. Secondo Lombroso (e numerosi altri medici e scienziati) i ribelli sono in fondo bestie, bruti privi di autentica dignità umana, intrinsecamente barbari e violenti: come si fa con gli animali impazziti, quando si sottraggono al lavoro cui sono sottoposti, o peggio ancora diventano rabbiosi o pericolosi, si deve abbatterli, per impedire che devastino tutto o uccidano chi incontrino sulla loro strada. Anche Zola è disgustato da quanto descrive, ma non condivide affatto l'idea dei suoi personaggi borghesi, secondo cui, per evitare l'apocalisse rivoluzionaria si deve far ricorso (e al più presto) all'esercito. A suo giudizio, quei demoni che si sono scatenati sono uomini disperati, non animali feroci privi di senno e di razionalità.
} 
economiche della borghesia e un modello bottom-up, basato sulle esigenze dei poveri. Fa da sfondo alla sua opera la rappresentazione ideologica di una borghesia industriale e progressista in cui un ruolo fondamentale è giocato dalla libera volontà degli attori che operano sul mercato. L’idea è che l'apertura al volontarismo possa trasformare la società e riassorbire la povertà, incoraggiando i capitali e favorendo gli investimenti. In questo clima - un clima di fiducia intorno al ruolo delle banche come strumento per rilanciare l'economia - nasce e si consolida il progetto di ristrutturazione di Parigi dell'architetto Haussmann, poi destinato a naufragare in speculazioni e corruzione. Ma è anche in questo clima che, da un lato, matura la legge sull'espro-priazione per pubblica utilità. Dall'altro, l'esigenza di protezione dalle masse povere, conduce alla "soluzione" dei Boulevard, ampi, larghi, dove è impossibile erigere barricate e più facile controllare le masse. Architettura che si piega all'esigenza dell'ordine pubblico e della sicurezza militare, più che a migliorare le condizioni di vita.

Il J'Accuse di Zola (1995) è protesta infiammata, grido dell'anima che attraverso il dramma di Alfred Dreyfus racconta, nel tentativo di sottrarre la giovane Repubblica a una fine precoce, l'agonizzante democrazia francese, vessata da guerre tra poteri e preda di corruzione diffusa ${ }^{8}$. Un'immagine apocalittica, al pari della società del Secondo Impero, analizzata nella Bestia Umana attraverso i personaggi di Madame Bonnehon, il Presidente Grandmorin, il ministro della Giustizia Camy-Lamotte e il giudice Monsier Denizet, anch'essi portatori di un mondo fatto di cinismo, opportunismo e false apparenze, contro ogni etica e morale: "Poi, mio Dio, la giustizia che ultima illusione! Voler essere giusto non è forse una chimera, quando la verità è in mezzo ai rovi?” (1981, p. 205).

\footnotetext{
"Ed è volontariamente che mi espongo. Quanto alle persone che accuso, non le conosco, non le ho mai viste, e non nutro contro di esse né rancore né odio. Per me sono soltanto entità e spiriti di malvagità sociale. E l'atto che compio oggi non è che un mezzo rivoluzionario per sollecitare l'esplosione della verità e della giustizia. Non ho che una passione, quella della chiarezza, in nome dell'umanità che ha tanto sofferto e che ha diritto ad essere felice" (Zola, 1995, p. 1). Nel suo celebre J'accuse Zola punta il dito contro tutti i responsabili di una delle pagine più cupe e miserabili della democrazia francese, la condanna di Alfred Dreyfus. Innocente, Dreyfus era un ufficiale ebreo impiegato presso il ministero della Guerra, accusato nel 1894 di aver rivelato importanti segreti militari relativi alla difesa nazionale all'addetto militare tedesco a Parigi. Una montatura. Nulla di vero.
} 
Il sottile filo che lega Zola a Kafka è nella descrizione di una società in cui agli individui non è concesso realizzare le proprie aspirazioni. Si è piuttosto in presenza di una società espressione di conformismo e ordinarietà. La crescente concentrazione della popolazione nella città (che a Parigi porta a sospendere i lavori di ristrutturazione), assume in Kafka la stessa carica negativa. In questo contesto, la descrizione della modernità di Kafka non lascia spazio alle relazioni interpersonali (che si deteriorano). La stessa cosa in Zola. In entrambi gli autori, il linguaggio è percepito come strumento di comunicazione. Esso presenta però dei limiti. La battaglia dello scrittore è quella di afferrare la verità e assicurare la comprensione. Più lo scrittore dettaglia il suo linguaggio per rendere efficace la descrizione per cogliere la complessità della realtà sociale e più il linguaggio appare inadeguato.

Ma in Zola il discorso si ferma alle soglie della vulnerabilità sociale. In Kafka il deterioramento delle relazioni umane apre alla persecuzione e allo sfruttamento perpetrato dalla giustizia. Non è un caso che, collocando il Tribunale in uno dei quartieri più poveri della città, il Processo (Kafka, 1969c) mette in evidenza come la realizzazione della conformità sociale passi attraverso la negazione dell'individuo.

Una catena ininterrotta di errori giudiziari, di ingiusti processi, di violenze carcerarie e di umane sofferenze lega epoche diverse e regimi diversi, fino ad imprigionare l'intera società organizzata in un sistema incapace di rinnovarsi e di garantire a tutti una giustizia giusta e una libertà inviolabile. Lo scontro drammatico fra modernità e burocrazia si fa, in Kafka, scontro fra Gesellschaft e Gemeinschaft. Il diritto, espressione della Gesellschaft, è frammentario, paradossale, contraddittorio.

3. E' ciò che accade a Joseph K., eroe del surreale immaginario kafkiano. Tra il primo e il decimo capitolo, rispettivamente polo della commedia e della tragedia, si dipana dolorosamente il processo. Vera via crucis che $\mathrm{K}$. affronterà dapprima con baldanza. La reazione innanzi al giudice istruttore è veemente: protesta, si indigna, strappa il quadernetto dalle mani del giudice. "Farabutti, teneteli per voi i vostri interrogatori" (Kafka, 1969c, p. 82), è la replica sdegnata con cui K. abbandona la sala 
d'udienza9. Poi con smarrito sospetto, fino alla più completa e debellata rassegnazione ${ }^{10}$. Egli sembra essere sottoposto a due differenti tipologie di giudizi. Come nel caso del Diritto civile e del diritto canonico, entrambi hanno base nel Corpus iuris, eppure sviluppano procedure che tradiscono la loro vicinanza.

Nel Processo la colpa è prodotta dal Tribunale, è una creazione arbitraria e sovrana dell'autorità, invece di essere scoperta e provata. Le caratteristiche del processo nel quale $\mathrm{K}$. è coinvolto ricalcano in modo interessante la legislazione penale austro-ungarica in vigore al tempo in cui Kafka scrisse. La legislazione disciplinava un processo inquisitorio, segreto e inaccessibile alle parti. Da qui l'autore trae ampi spunti per descrivere l'enigmatico caso di Joseph K. Tanto nel Processo quanto nel racconto Nella colonia penale ${ }^{11}$ (Kafka, 1992e), egli esplora il tema della colpa

9 Con un qualche fondamento Cavallone (2002, p. 586) osserva che K. dà l'impressione di potersi liberare del processo semplicemente rigettandolo con un gesto di volizione psicologica; ciononostante, "l'infezione procede inesorabile" come fosse una "sindrome ossessiva" dalla quale si muore prescindendo da quella che sarà la conclusione del processo stesso. "Il pensiero del processo non l'abbandona più", si dice all'inizio del settimo capitolo, "ed è questa la vera malattia epidemica" (Cavallone, 2002, p. 588). Secondo Citati, "come l'ultimo carcerato, vive soltanto nella dimensione del processo, incapace di cancellare dalla mente il pensiero di quella mattina - gli sconosciuti nella sua stanza, l'aggressione nella sua casa -: mentre se sapesse dimenticare, le guardie, i giudici e le gerarchie del Tribunale ritornerebbero forse nel vuoto dal quale sono usciti" (2007, p. 168).

10 K. capirà a poco a poco che lui stesso è divenuto parte del processo, che è il processo ad essersi impadronito di lui, a contagiarlo (privandolo di qualsiasi dignità). Il processo è la perdita di fiducia, l'abbandono di qualsiasi speranza, il consegnarsi senza più combattere a chi, in silenzio "ti farà la pelle" (Capponi, 2013, p. 541). Uno dei più autorevoli traduttori di Kafka, Primo Levi, ha scritto che la lettura del romanzo "lascia mutati: più tristi e più consapevoli di prima [...] tradurre è più che leggere: da questa traduzione sono uscito come da una malattia" (Cavallone, 2002, p. 589). Ed aggiunge Cavallone: "forse il virus del processo è un ceppo unitario, dal quale però traggono origine specie diverse a seconda dei climi sociali e culturali" (2002, p. 589); ed ancora "il Tribunale K. lo ha interiorizzato, metabolizzato, se lo porta dentro ovunque vada" (2002, p. 586).

"Nella colonia penale è il racconto in cui Kafka esplora il difficile rapporto tra giustizia e punizione; è una narrazione che sovverte i principi cardine del diritto penale moderno: legalità, colpevolezza e giusto processo. Quattro sono i personaggi della storia, che si svolge in una vallata deserta e desolata: l'esploratore, l'ufficiale, il soldato e il condannato. Quest'ultimo non sa di esserlo né ha idea della pena che sta per essergli inflitta. L'esploratore trova ripugnante il fatto che al condannato non sia stata data la possibilità di conoscere l'accusa e di difendersi, ma l'ufficiale gli spiega perché questo modo di procedere non è sbagliato. Di fronte al principio di giustizia adottato nella colonia penale, secondo cui la colpevolezza è sempre presunta e non può essere messa in dubbio, non c'è spazio per la difesa e quindi per il processo. Più che garantire un giusto processo all'imputato è necessario assicurare l'efficienza del sistema: se l'imputato fosse messo a conoscenza dell'accusa, occorrerebbe discutere della sua asserita innocenza, ostacolando il corso della giustizia. In questo modo, peraltro, l’imputato non può capire la sua colpa, come del resto non comprende nulla di quanto accade intorno a lui, dal momento che parla una lingua diversa da quella dell'esploratore. E' necessario lasciare spazio quanto 
oggettiva, scevra di qualunque riferimento fattuale e soggettivo. Una volta avviato un procedimento nei confronti di una persona, questa è colpevole per il fatto stesso di essere stata sottoposta a processo e non v'è possibilità che il tribunale kafkiano cambi idea e allenti la sua presa soffocante sul malcapitato imputato, completamente ipnotizzato ed assorbito, giorno dopo giorno, entro quell'inestricabile, asfissiante, farraginoso labirinto che è il tribunale. "Paese dello spazio indefinito"12, dove "anche il suddito è straniero"13 (Cacciari, 1985, p. 115).

Ecco il leitmotiv, la chiave dell'argomentare kafkiano in tutte le sue opere, sia nel Processo (Kafka, 1969c), sia nella Metamorfosi (Kafka, 1992c), sia nel Castello (Kafka, 1969b): l'organizzazione giudiziaria, che occupa guardiani corruttibili, ispettori straccioni, modesti giudici istruttori,

prima alla condanna, che verrà inflitta attraverso un terribile e gigantesco macchinario con il quale verrà tatuato profondamente sul corpo del condannato il comando che ha violato. Leggendolo sul suo corpo, questi potrà comprendere l'errore commesso.

${ }_{12}$ Nel "Paese dallo spazio indefinito" il diritto è sradicato, cioè "letteralmente, desacralizzato", e, come tale, despazializzato, trascina via dal proprio posto per collocarci in un non-posto. Sullo sradicamento del Nomos, cfr. M. Cacciari, Geofilosofia dell'Europa, de M. Cacciari (1994). Sui processi di "sconfinamento" del diritto attuale, tipici della trasnazionalizzazione e globalizzazione giuridica, cfr. M. R. Ferrarese, Diritto sconfinato. Inventiva giuridica e spazi nel mondo globale, Roma-Bari, 2006; S. Cassese, Oltre lo Stato, Roma-Bari, 2006; U. Vincenti, Diritto senza identità. La crisi delle categorie giuridiche tradizionali, Roma-Bari, 2007. Sulla dimensione globale del diritto come artificialità, che governerebbe gli spazi lasciati vuoti dallo sradicamento del Nomos ed occupati dal "mercato", cfr. N. Irti, Norma e luoghi Problemi di geo-diritto, Roma-Bari, 2001; Id., Il salvagente della forma, Roma-Bari, 2007. "In che villaggio mi sono smarrito? C'è un Castello qui?", chiede K. rivolto all'oste e al giovanotto in abito cittadino che ha incontrato nell'osteria (Kafka, 1969b, p. 564). Allo stesso modo, il protagonista di un altro romanzo kafkiano si trova sin da subito smarrito: pur avendo già da tempo avvistato la Statua della Libertà dalla nave che sta per far ingresso nel porto di New York, il giovane Karl Rossmann, pronto a sbarcare ma costretto a tornare sui suoi passi per cercare l'ombrello che ha dimenticato, finisce per perdersi proprio all'interno della nave su cui aveva viaggiato. "Mi sono sperduto [...] durante il viaggio non me n'ero veramente accorto, ma questa nave è terribilmente grande", dice Karl all'uomo gigantesco che si trovava dietro la "porticina" contro cui si era accanito a battere col pugno (Kafka, 1969a, p. 3-4). Non diversa sorta tocca al protagonista del Processo, quel Joseph K. che una mattina, al risveglio, si ritrova straniero in casa propria (Kafka, processo, 1969, p. 921). L'incipit di tutti e tre i romanzi rivela questa dimensione del dis-orientamento, della dispersione. K., Karl e Joseph K. sono dipendenti dal fattore dis-orientamento, così come i loro nomi dipendono tutti da una sola lettera: la lettera "K", il marchio di fabbrica che li contraddistingue.

${ }_{33}$ Belloni (2009, p. 236) afferma in punto: "è questo lo spazio in cui si smarriscono i personaggi kafkiani, il portato della crisi irreversibile del Nomos, così ben visibile in quell'opera di costruzione della Muraglia cinese a cui si riferisce Kafka in un suo celebre racconto (Kafka, 1992b, p. 398-412) [...] La Muraglia di cui parla Kafka si costruisce per grossi buchi e non è che terminata nel suo tratto più settentrionale. Il suo essere ancora in costruzione suona più che alto come la fine della sua costruzione, ovvero come l'impossibilità di radicarla. Altrettanto irrealizzabile sarà anche la Torre (Kafka, 1992d, p, 431-32), che non a caso [...] viene identificata come una "nuova Torre di Babele" e che per essere eretta avrebbe dovuto reggersi sulla Muraglia”. 
la cui finalità consiste nell'arrestare "innocenti", e agire nel più insensato dei modi ${ }^{14}$; la assoluta impossibilità di consultare gli atti del processo, che col processo nulla hanno a che vedere ${ }^{15}$. Si legge:

Non ho mai sentito di un'assoluzione vera, e ho saputo di molti giudici che sono stati influenzati. Devo ammetterlo, non ho mai assistito a un solo caso di assoluzione vera. D’altra parte, com'è possibile far capo a precedenti? Le sentenze definitive dei tribunali non vengono pubblicate, non sono accessibili neppure ai giudici, e perciò sui casi giudiziari del passato non si sono conservate che leggende (Kafka, 1969c, p. 126).

Mentre però nel Processo la dimensione è quella punitiva del diritto penale, ne Il castello la dimensione in cui Kafka si muove è piuttosto quella del diritto privato e il suo tono è conciliativo, tipico di una giustizia restitutiva. Il diritto di fonte statale non è coinvolto ne Il Castello, come lo è, invece, nel Processo. Il signore del Castello ha già condannato K. all'esilio, facendo applicazione di regole consuetudinarie. E. Durkheim, dal canto suo, aveva già evidenziato l'esistenza di diversi livelli di solidarietà sociale e coesione morale. Al differenziarsi della società, il diritto evolve da forme repressive (pene) a forme restitutive (compensation).

La "lotta" di K., la sua battaglia, nasconde allora il suo desiderio di vincere una burocrazia che, negando i suoi diritti, gli impedisce di entrare a far parte della Gemeinschaft. Nel Processo, invece, la legge si presenta come strumento organizzato della Gesellschaft.

\footnotetext{
${ }_{14}$ Tutte le componenti - giudici, avvocati, cancellieri,ufficiali giudiziari e quanti altri si aggiungano alla bisogna - svolgono funzioni che assicurino il continuo movimento del'organizzazione; di qui, contraddizioni, illogicità: non è dato conoscere né le accelerazioni, né le soste, né meno che mai il suo quietarsi (Salazar, 2005, p. 371). Se questo è il processo, cosa è la giustizia? Coerentemente non ci si può attendere che essa non si regga sulla aleatorietà, l'arbitrarietà, la illogicità. L'avvocato deve informare il cliente di come opera il sistema giudiziario, senza illuderlo di giustizia perché quel sistema ha la sola finalità di far funzionare la macchina, senza preoccuparsi dell'imputato che per sua disgrazia si dovesse rivolgere al Tribunale "entità misteriosa, segreta e manifesta, celata e apparente, invisibile e visibilissima" (Citati, 2007, p. 127).

${ }_{15}$ Non v'è certezza, nel ritratto kafkiano, che gli atti siano esaminati, addirittura rintracciati e non smarriti (come spesso accade oggi in certi tribunali, per cui lo spostamento di un fascicolo da una casella all'altra della cancelleria ostacola il corso di un processo per anni). D'altronde, in un processo così immaginato, in cui il valore della difesa non esiste, sostituito dal valore di far funzionare la macchina organizzativa, cosa serve l'esame degli atti? Cosa serve motivare la condanna? Cosa serve assolvere o condannare l'imputato? Salazar (2005, p. 376) ricorda la sentenza n. 27/2000 del Tribunale di Siena, annullata dalla Cassazione con sentenza del 2 luglio 2004, n. 12.114, perché incomprensibile, scevra di ogni possibile senso, recte priva di valida motivazione.
} 
La drammatica esperienza e l'impossibilità di seguire lo svolgimento del processo lasciano intendere che nessuno possa ottenere giustizia16.

Emblematico è il ritratto della Giustizia, commissionato al pittore giudiziario Titorelli: essa è raffigurata con la benda sugli occhi, la bilancia tra le mani e le ali ai calcagni, nell'atto di correre. Si tratterebbe della Giustizia e della Vittoria, insieme. E tuttavia K. osserva, perplesso, che "non è una buona combinazione. La Giustizia deve star ferma, altrimenti la Bilancia si muove e non è possibile pronunciare una sentenza giusta" (Kafka, 1969c, p. 86). Più che la Dea della Giustizia e della Vittoria, la figura de qua appare più ricordare la Dea della Caccia: la giustizia kafkiana è cacciatrice, 'stana' le colpe di persone che pure non sanno di averle e, una volta avviato il processo, questo non può che concludersi con una condanna, perché la colpa è in re ipsa. Ad essa farà sempre seguito una punizione inevitabile inflitta da una Giustizia incomprensibile, inaccessibile e cacciatrice.

La legge innanzi alla quale vengono a trovarsi Joseph K. e l'uomo di campagna della parabola ${ }^{17}$ è muta e incomprensibile . Di fronte a questo perdurante silenzio, entrambi i protagonisti kafkiani sono costretti a vivere un'attesa indefinita e paralizzante. L'incapacità dell'uomo di campagna di superare il guardiano, che impersona il diritto vivente ${ }^{18}$, e l'incapacità di $\mathrm{K}$.

\footnotetext{
${ }_{16}$ Neppure al momento del tragico epilogo, prima di essere giustiziato, vengono sciolti gli enigmi del processo: "C'erano obiezioni dimenticate? Certo che ce n'erano. La logica è bensì incrollabile, ma non resiste a un uomo che vuol vivere. Dov'era il giudice che egli non aveva mai visto? Dove il supremo tribunale fino al quale non era mai arrivato?” (Kafka, 1969c, p. 532).

${ }_{17}$ Il racconto è ben noto: narra la storia di un "uomo di campagna" che, arrivato alle porte della legge, si scontra con l'opposizione di un guardiano che gli impedisce di entrare. Pazienta per anni ed anni, urtandosi al medesimo rifiuto ogni volta che rinnova la sua richiesta. Infine, logorato ed invecchiato, si stupisce di essere da così tanto tempo il solo a reclamare l'accesso alla legge, e ottiene la seguente risposta: "Nessun altro poteva entrare qui perché questo ingresso era destinato soltanto a te. Ora vado a chiuderlo" (Kafka, 1969c, p. 178). Ne va dunque dell'accessibilità della legge (o piuttosto della sua inaccessibilità, del mistero o dell'opacità della sua origine), come J. Derrida (1985; 1994; 1996; 2003) non manca di ricordare. Questa breve parabola di due pagine può essere considerata una sintesi della posta in gioco principale del Processo. L'autore stesso ha d'altronde estratto Vor dem Gesetz dal manoscritto del suo romanzo per pubblicarlo separatamente, il 7 settembre 1915, nel numero Nouvel An Juif della rivista Sembswehr (n. 24).

${ }_{18}$ Il tempo della legge è il tempo dell'attesa: è attesa concreta, burocratica, che blocca i soggetti, impantanati nell'illusione di chiedere ed ottenere giustizia, ovvero risposte, garanzie, diritti, certezze, come è per l'uomo di campagna del racconto Davanti alla legge (Kafka, 1992a), che rimane seduto davanti alla porta della legge "per giorni e anni. Fa numerosi tentativi per passare e stanca il guardiano con le sue richieste" (Kafka, 1992a, p. 75), fino al termine della sua esistenza, quando all'illusione di poter varcare la soglia della
} 
di scoprire l'accusa rivolta contro di lui è la metafora della difficoltà dell'uomo comune di capire la legge e i suoi meccanismi e dell'angoscia che nel processo attanaglia le parti che si scoprono impotenti rispetto al loro stesso destino. Il campagnolo pensava ingenuamente che la legge dovesse "essere accessibile a tutti e in ogni momento" (Kafka, 1969c, p. 175), e allo stesso modo K. pensa di poter essere ascoltato, si illude che la sua istanza possa favorirgli l'accesso al Tribunale, si illude di poter essere ammesso a discutere delle sue presunte colpe. E', infine, la metafora dell'incolmabile dicotomia che esiste tra le aspettative di giustizia, e la sua tensione all'universalità, dell'uomo comune e i risvolti effettivi del processo, vale a dire il riconoscimento della singolarità e specificità della giustizia.

Joseph K., out-law peculiare, giacché costantemente anelante alla Legge, sorretto da una "furia interpretativa" (Anders, 1989), ossessionato dall'agire adempiente-obbediente (l'Achtung di kantiana memoria), dal dovere per il dovere, dopo aver lottato, si troverà ad accettare una condanna senza giudizio. È questo il fardello dei personaggi kafkiani: la crescente angoscia derivante dal non poter essere assolti (non vale il rinvio, non l'assoluzione provvisoria) e dal non potersi purgare agli occhi del mondo, che già li ha giudicati anche nella superfluità del giudizio stesso. Il mite bancario di mezza età, prototipo del meteco, dello straniero-interno, capirà poco a poco, come lui stesso sia divenuto parte del processo, come sia stato il processo ad essersi impadronito di lui, ad averlo "contagiato", privandolo di qualsiasi dignità, "disumanizzandolo"19. La stessa disumanizzazione dell'immondo insetto Gregor Samsa, uomo racchiuso in un sistema familiare e sociale sordido e moralistico, "condannato a lavorare in una ditta, presso la quale la più piccola trascuratezza provocava il maggior sospetto" (Kafka, 1992c, p. 127).

Il processo è la perdita di fiducia, l'abbandono di qualsiasi speranza, il consegnarsi senza più combattere a chi, in silenzio, eseguirà la propria

legge sembra aggiungersi quella, alimentata dal guardiano stesso, di un ingresso desinato esclusivamente a lui. Questa illusione pare caratterizzare sempre più la nostra dimensione giuridica contemporanea e riguardare proprio quelle categorie di soggetti sempre più ultimi, precarizzati e marginalizzati che vivono sulla "soglia" di molte delle società attuali (Dal Lago, 1999).

19 Secondo Cavallone: "il Tribunale K. lo ha interiorizzato, metabolizzato, se lo porta dentro ovunque vada” (2002, p. 586). 
condanna, una condanna senza appello, in nome di un diritto ormai "svuotato", desacralizzato, ridotto a mera usanza ed abitudine, totalmente privo della dimensione dell' $i$, della giustizia ${ }^{20}$, "slegato dalla Tessitura cosmica, dimentico del Tao celeste e non più in accordo con la sua caratterizzazione mitica ed originaria" (Belloni, 2009, p. 238).

Il dramma di K., rimanda al più doloroso mistero della cultura cristiana: un'accusa ingiusta, un processo sommario, un atto di clemenza negato, un'uccisione rituale. Il Golgota è qui una squallida cava, la croce un affilato coltello da norcino; ma nella passione e nella morte non c'è riscatto, non c'è liberazione, non ci sarà redenzione. Fino all'ultimo K., cui la vita non ha riconosciuto la dignità di un processo celebrato in una sede naturale e in una forma legale, spalancandogli la via di una condanna senza giudizio, $\mathrm{o}$ addirittura, di un giudizio giudicato superfluo, spererà nell'aiuto di qualcuno, fosse anche l'estraneo che s'affaccia neghittosamente alla sua finestra sulla cava, mentre la voce narrante continua a domandarsi con angoscia crescente: "un aiuto era ancora possibile? C'erano eccezioni non sollevate per negligenza? Dov'era il giudice, che lui non aveva mai visto? Dov'era l'Alta Corte, davanti a cui non era mai giunto?” (Kafka, 1969c, p. 187).

Il protagonista K. non conosce il reato per cui è imputato, e il suo giudice è invisibile e inavvicinabile. Metafisica, fantasia letteraria, profezia delle brutalità imminenti? Forse non del tutto, perché - scrive De Cataldo - "aspetti che definiremmo oggi tipicamente kafkiani sono rimasti a lungo presenti nei codici di procedura anche delle più avanzate democrazie" (2010, p. 6). Basti pensare ad esempi attuali e tristemente famosi: il carcere di Guantanamo, i casi di "rendition" (i sequestri in territorio estero avallati dagli Stati), i centri di permanenza per i migranti, vere e proprie prigioni senza processo.

Nel fluire del racconto fantastico, dunque, sono presenti molti aspetti di realtà che nel tempo non sembrano mutati: imprevedibilità $\mathrm{e}$ l’incontrollabilità dei giudizi; l'inat-tendibilità dei magistrati, conformisti e

\footnotetext{
2o Per le dimensioni della giuridicità (il $l i$, il $f a$, l'i), tipiche del mondo culturale cinese, e su come esse giochino un ruolo al'interno dell'opera kafkiana, si fa riferimento agli studi di Cacciari (1985), Andreozzi (1878), Couvreur (Li Ki, 1899), Van Der Sprenkel (1966), Lanciotti (1978), Benecke (1989-1992) e Huisman e Malafray (1992).
} 
pigri; l'ignoranza verbosa degli avvocati, che tuttora amano distinguersi in "grandi" e "mestieranti", perdendo di vista la dignità della professione; la difficoltà di conoscere i giudicati, grazie alle modalità sincopate di redazione delle sentenze; l'impossibilità di conoscere la legge, e quindi di rispettarla; il difficile dialogo col giudice, tra udienze inutili, protocolli rituali, scambi di memorie non lette; l'inanità dell'individuo dinanzi alla legge ed al processo; il doversi piegare alla logica comune, pur allorquando non sia possibile condividerla; i meriti che all'individuo vengono riconosciuti non per qualità personali, ma per la sua appartenenza ad un gruppo, per l'ascendenza familiare (come nel caso del ritrattista giudiziario), per convenzione sociale, per la diseguaglianza tra uomini che pur amano farisaicamente definirsi tra loro eguali; il dover chiedere come un favore capricciosamente accordato ciò che invece spetta di diritto; l'attesa che la giustizia sia finalmente resa, mentre nel vuoto il giudizio degli altri si diffonde e si consolida prescindendo da quello della corte.

La testimonianza letteraria, in conclusione, aiuta a capire che la verità giudiziaria non è l'unica possibile (Marra, 2010; Sansone, 2001; Mittica, 2009). Che essa va collocata in un mondo di relazioni in cui le azioni degli uomini siano restituite alla loro complessità. Dal punto di vista della comunità siamo legati da una lege più profonda di quella giuridica - che la integra senza identificarsi con essa. Anche su questa consapevolezza poggia il ponte invisibile che congiunge le sponde opposte della letteratura e del diritto. Condivisibile è allora l'osservazione, espressa nella prefazione alla recente pubblicazione nella sua versione integrale dell'Affare Dreyfus, dal giornalista-scrittore Saviano:

Nei momenti insopportabili del quotidiano quando le notizie ti raggiungono come prova oggettiva dell'impossibilità di poter vivere in un paese giusto, quando ti accorgi che la soluzione adottata dai più è di abbandonarsi al livore o alla rassegnazione, ci sono pensieri che riescono a concedere una possibilità di soluzione. Qualcosa in più di un semplice conforto (2012, p. II). 
La letteratura fornisce spunti e delinea percorsi per immaginare una "Nuova Giustizia”21.

\section{RIFERIMENTI}

ANDERS, G. Kafka. Pro e contro. I documenti del processo. Ferrara: G. Corbo, 1989.

ANDREOZZI, A. Le leggi penali degli antichi cinesi. Discorso proemiale sul diritto e sui limiti del punire, e traduzioni originali dal cinese. Firenze, 1878.

BELLONI, I. Il fattore K. Legge, vita, corpo nell'opera di Franz Kafka. Annali-Università degli studi Suor Orsola Benincasa, n. 1, p. 233-26o, 2009.

BENECKE, G. The comparative history of custom in chinese law. Recueils de la Société Jean Bodin, v. 53, pp. 427-448, 1989-1992;

BERGSON, Henri-Louis. Les deux sources de la morale et de la religion. Paris: PUF, 1932.

CACCIARI, M. Icone della legge. Milano: Adelphi, 1985.

CACCIARI, M. Geofilosofia dell'Europa. Milano: Adelphi, 1994.

CALAMANDREI, P. Le lettere e il processo civile. Rivista di dir. proc. civile, I, 1924 .

CAMERANA, O. La giustizia e le sue chiavi perdute. L'osservatore romano, Città del Vaticano, p. 5, 11-12 marzo 2013.

CARDOZO, B. N. Law and Literature. Yale Review, v. 14, p. 699-718, 19241925 .

CASSESE, S. Oltre lo Stato. Roma; Bari: Laterza, 2006.

CAVALLONE, B. Il processo come contagio. Rivista di diritto processuale, n. 2, p. 581-594, 2002.

CITATI, P. Kafka. Milano: Adelphi, 2007.

DAL LAGO, A. Non-persone. L'esclusione dei migranti in una società globale. Milano: Feltrinelli, 1999.

DANOVI, R. Recensione di «Giustizia e Letteratura I-II», a cura di G. Forti, C. Mazzucato, A. Visconti. La Previdenza forense, n. 2, p. 190-191, 2014.

DE CATALDO, Giancarlo. Introduzione. In: KAFKA, F. Il processo. Roma: Newton Compton, 2010.

DERRIDA, J. Préjugés. «Devant la loi». In: AA.VV. La faculté de juger, colloque de Cerisy. Paris: Les éditions de Minuit, 1985.

\footnotetext{
${ }_{21}^{21}$ L'ingiustizia, del resto, è stato detto, "è la cosa più atroce di questo mondo; non posso descriverti come mi sento quando mi trovo di fronte all'ingiustizia, non importa se fatta a me o ad altri; mi passa da parte a parte, mi dolgono il corpo e l'anima, mi pare che mi si colmi la bocca di sabbia e che io debba soffocare lì per lì” (Wassermann, 2007, p. 72).
} 
DERRIDA, J. Force del loi. Le "Fondament mystique de l'autorité". Paris: Galileé, 1994.

DERRIDA, J. Pre-giudicati. «Davanti alla legge». Catanzaro: Abramo, 1996.

DERRIDA, J. Forza di legge. Trad. di A. Di Natale. Torino: Bollati Boringhier, 2003.

FERRARESE, M. R. Diritto sconfinato. Inventiva giuridica e spazi nel mondo globale. Roma-Bari: Laterza, 2006.

FORTI, G.; MAZZUCATO, C.; VISCONTI, A. Giustizia e Letteratura I. Milano: Vita e Pensiero, 2012.

FORTI, G.; MAZZUCATO, C.; VISCONTI, A. Giustizia e Letteratura II. Milano: Vita e Pensiero, 2014.

HUISMAN, D.; MALAFRAY, M. A. Les plus grands textes de la philosophie oriental. Paris: Albin Michel, 1992.

IRTI, N. Norma e luoghi. Problemi di geo-diritto. Roma; Bari: Laterza, 2001.

IRTI, N. Il salvagente della forma. Roma; Bari: Laterza, 2007.

KAFKA, F. America. In: KAFKA, F. Romanzi. Trad. A. Rho. Milano: Mondadori, 1969a.

KAFKA, F. Il castello. In: KAFKA, F. Romanzi. Trad. A. Rho. Milano: Mondadori, 1969b.

KAFKA, F. Il processo. In: KAFKA, F. Romanzi. Trad. A. Rho. Milano: Mondadori, 1969c.

KAFKA, F. Davanti alla legge. In: KAFKA, F. Racconti. Milano: Mondadori, 1992a.

KAFKA, F. Durante la costruzione della muraglia cinese. In: KAFKA, F. Racconti. Milano: Mondadori, 1992b.

KAFKA, F. La metamorfosi. In: KAFKA, F. Racconti. Milano: Mondadori, $1992 \mathrm{C}$.

KAFKA, F. Lo stemma cittadino. In: KAFKA, F. Racconti. Milano: Mondadori, $1992 d$.

KAFKA, F. Nella colonia penale. In: KAFKA, F. Racconti. Milano: Mondadori, 1992e.

LANCIOTTI, L. (Org.). Il diritto in Cina. Teoria e applicazioni durante le dinastie imperiali e problematica del diritto cinese contemporâneo. Atti del Convegno Internazionale di Studi Cinesi, Venezia, 14-15 ottobre 1976. Firenze: Olschki, 1978.

KI, Li. Mémoires sur les bienséances et les cérémonies. Trad. S. Couvreur. Paris: Ho Kien Fu; Imprimerie de la Mission Catholique, 1899. v. 2.

MAETERLINCK, M. Il tesoro degli umili. Palermo: La Zisa, 1989.

MAGRIS, C. Davanti alla legge. Trieste: EUT, 2006. 
MARRA, R. Dei diritti e delle pene: tra letteratura e diritto. La cognizione del delitto. Reato e "macchina della giustizia" nel Pasticciaccio di Gadda. Materiali per una storia della cultura giuridica, v. 40, n. 1, p.157-186, 2010.

MITTICA, M. P. Diritto e letteratura in Italia. Stato dell'arte e riflessioni sul metodo. Materiali per una storia della cultura giuridica, anno 39, n. 1, p. 273-299, 2009.

PERGOLESI, F. Il diritto nella letteratura. Archivio Giuridico "Filippo Serafini”, v. XCVII, fasc. 1, p. 61-104, 1927.

PERGOLESI, F. Diritto e giustizia nella letteratura moderna narrativa e teatrale. Bologna: Zuffi, 1956.

SALAZAR, M. L'avvocato di carta. Milano: Giuffrè, 2005.

SANSONE, A. Diritto e letteratura. Milano: Giuffrè, 2001.

SATTA, S. Il mistero del processo. In: SATTA, S. Soliloqui e colloqui di un giurista. Padova: Cedam, 1968.

SAVIANO, R. L'affaire Dreyfus: La verità in cammino. Firenze: Giuntina, 2012.

TOBAGI, B. Come mi batte forte il tuo cuore. Storia di mio padre. Torino: Einaudi, 2009.

TUMIATI, L. La poesia nel diritto. Rivista internazionale di filosofia del diritto, v. III, p. 261-272, 1927.

VACCA, R. Il diritto sperimentale. Torino: Fratelli Bocca, 1923.

VAN DER SPRENKEL, S. Legal institutions in Manchu China. A sociological analysis. London: Athlone Press, 1966.

WASSERMANN, J. Il caso Maurizius. Roma: Newton Compton, 2007.

WIGMORE, J. A List of Legal Novels. Illinois Law Review, v. 2, p. 574-593, 1908.

ZOLA, E. Bestia umana Milano: Rizzoli, 1981.

ZOLA, E. J’accuse. Milano: Mondadori, 1995.

ZOLA, E. Germinale. Torino: Einaudi, 2005.

ZOLA, E. L'assommoir. Milano: Rizzoli, 2009a.

ZOLA, E. Teresa Raquin. Milano: Mondadori, 2009b.

Lingua originale: Italiano

Ricevuto: 01/03/15

Accettato: 24/07/15 